\title{
Indoleamine 2,3-dioxygenase downregulates T-cell receptor complex $\zeta$-chain and c-Myc, and reduces proliferation, lactate dehydrogenase levels and mitochondrial glutaminase in human T-cells
}

\author{
THEODOROS ELEFTHERIADIS, GEORGIOS PISSAS, GEORGIA ANTONIADI, KONSTANTINA TSOGKA, \\ MARIA SOUNIDAKI, VASSILIOS LIAKOPOULOS and IOANNIS STEFANIDIS
}

Department of Nephrology, Medical School, University of Thessaly, Larissa 41110, Greece

Received January 12, 2015; Accepted October 19, 2015

DOI: $10.3892 / \mathrm{mmr} .2015 .4595$

\begin{abstract}
Indoleamine 2,3-dioxygenase (IDO), through L-tryptophan depletion, activates general control non-derepressible (GCN) 2 kinase and suppresses T-cell proliferation, in addition to suppressing aerobic glycolysis and glutaminolysis, which are required for these rapidly proliferating cells. A number of, however not all of these alterations, are partially mediated through IDO-induced p53 upregulation. In two-way mixed lymphocyte reactions (MLRs), IDO reduced cellular proliferation. In MLR-derived T-cells, IDO induced the expression levels of p53 and p21, however concurrently reduced the levels of $\zeta$-chain, c-Myc, lactate dehydrogenase A (LDH-A) and glutaminase (GLS)2. However, p53 had no effect on the expression of the above proteins. These results were recapitulated in T-cells activated with anti-CD2, anti-CD3 and anti-CD28 by direct activation of the GCN2 kinase with tryptophanol. In conclusion, IDO, through GCN2 kinase activation, downregulates the levels of TCR-complex $\zeta$-chain and c-Myc, resulting in the suppression of T-cell proliferation and a reduction in the levels of LDH-A and GLS2, which are key enzymes involved in aerobic glycolysis and glutaminolysis, respectively.
\end{abstract}

\section{Introduction}

Indoleamine 2,3-dioxygenase (IDO) degrades L-tryptophan via the kynurenine pathway. L-tryptophan depletion activates general control non-derepressible (GCN)2 kinase, which phosphorylates eukaryotic initiation factor $2 \alpha$, altering the translation program of T-cells and leading to the inhibition of cellular proliferation and anergy (1). An additional pathway able

Correspondence to: Professor Theodoros Eleftheriadis, Department of Nephrology, Medical School, University of Thessaly, Neo Ktirio, Mezourlo Hill, Larissa 41110, Greece

E-mail: teleftheriadis@yahoo.com

Key words: indoleamine 2,3-dioxygenase, T-cells, ל-chain, c-Myc, aerobic glycolysis, glutaminolysis to sense amino acid deprivation is the mammalian target of rapamycin complex (mTORC)1 pathway (2).

Under inflammatory conditions IDO is upregulated in antigen presenting cells (APCs), including monocytes, macrophages and dendritic cells, and restricts the T-cell response $(3,4)$. Expression of IDO in APCs reduces graft rejection (5-7) and ameliorates autoimmune diseases (8-10). In addition, IDO is expressed in certain non-immune cells. Expression of IDO in a paternally derived placental trophoblast is required for a successful semi-allogenic pregnancy $(11,12)$, while expression in tumor cells contributes to their escape from immunosurveillance (13). Patients on hemodialysis are characterized by impaired adaptive immunity and exhibit increased expression of IDO, further enhanced in those who are non-responders to vaccination against hepatitis B virus (14). In these patients, plasma IDO levels are negatively associated with the T-cell count (15). Therefore, IDO is an enzyme, which serves an important role in immune system homeostasis and clarification of its mechanism of action may contribute to an improved understanding of immune system physiology, potentially leading to novel means of pharmaceutical intervention.

In previous studies, IDO-induced L-tryptophan depletion was demonstrated to activate the GCN2 kinase, whilst mTORC1 was unaffected in human alloreactive T-cells. In addition, in parallel with a reduction in T-cell proliferation, IDO reduced glucose consumption and lactate production by T-cells $(16,17)$. This indicates that IDO suppresses aerobic glycolysis in activated T-cells. The majority of rapidly proliferating cancer cells are characterized by an increased ratio of cytoplasmic glycolysis to mitochondrial glucose oxidation, a phenomenon first described by Otto Warburg, and hence termed the Warburg effect or aerobic glycolysis (18). Rapidly proliferating activated T-cells reprogram their metabolic pathways from pyruvate oxidation via the Krebs cycle to the glycolytic, pentose-phosphate and glutaminolytic pathways in order to fulfill the bioenergetic and biosynthetic demands of proliferation (19). Notably, IDO reveals no effect on the levels of activated pyruvate dehydrogenase or its inactive phosphorylated-Ser393 form, which controls the influx of pyruvate into the Krebs cycle (16).

A previous study demonstrated that in alloreactive T-cells, IDO increases the levels of p53, which contributes to 
the suppression of proliferation and aerobic glycolysis. The IDO-induced increase in p53 upregulated the expression levels of cyclin-dependent kinase inhibitor p21. IDO and p53 both reduced glucose consumption. The IDO-induced increase in p53 levels reduced the expression of glucose transporter-1, and increased the expression of TP53-induced glycolysis and apoptosis regulator, inhibiting glucose influx into T-cells and reducing glycolysis. However, IDO downregulated lactate dehydrogenase-A (LDH-A) and glutaminase (GLS)2, which are key enzymes in aerobic glycolysis and glutaminolysis, respectively, in a p53-independent manner. In addition, IDO and not p53 reduced lactate production (20).

As T-cell activation increases the transcription factor c-Myc, which subsequently upregulates LDH-A and GLS2 (19), in the present study the effect of IDO or direct GCN2 kinase activation on the expression levels of c-Myc, LDH-A and GLS2 in T-cells was investigated. In addition, the effect of IDO or direct GCN2 kinase activation on the expression of T-cell receptor (TCR)-complex $\zeta$-chain was investigated, as downregulation of this key molecule has been previously demonstrated to reduce the expression of c-Myc and T-cell proliferation (21).

\section{Materials and methods}

Subjects. Blood samples were collected from 10 non-related healthy volunteers (5 males and 5 females; age, 27-49 years). Informed consent was obtained from each individual enrolled in the study and the study protocol was approved by the by the ethics committee of the University Hospital of Larissa, Medical School, University of Thessaly (Larissa, Greece).

Peripheral blood mononuclear cell (PBMC), and T-cell isolation and culture. PBMCs were isolated from whole blood by Ficoll-Hypaque density gradient centrifugation (Histopaque 1077; Sigma-Aldrich, St. Louis, MO, USA) and quantified using an optical microscope (Axiovert $40 \mathrm{C}$; Carl Zeiss AG, Oberkochen, Germany) and a Neubauer chamber (Paul Marienfeld GmbH, Lauda-Königshofen, Germany). Cell viability was assessed by trypan blue staining (Sigma-Aldrich).

PBMCs were resuspended in RPMI-1640 medium containing L-glutamine and $10 \mathrm{mM}$ 4-(2-hydroxyethyl)-1-pip erazineethanesulfonic acid, and supplemented with $10 \%$ fetal bovine serum and antibiotic-antimycotic solution (dilution, 1:100) (all from Sigma-Aldrich).

For the experiments with the GCN2 kinase activator, tryptophanol (TRP; Sigma-Aldrich), T-cells were isolated from PBMCs using a Pan T-cell Isolation kit (Miltenyi Biotec $\mathrm{GmbH}$, Bergisch Gladbach, Germany). Non-T-cells were indirectly magnetically labeled and were depleted from the PBMC samples. Isolated T-cells were cultured in the same medium as the PBMCs. All cultures were incubated at $37^{\circ} \mathrm{C}$ in a humidified atmosphere containing $5 \% \mathrm{CO}_{2}$.

Assessment of cell proliferation in two-way mixed lymphocyte reactions. Two-way mixed lymphocyte reactions (MLRs) were performed in 96-well plates for 7 days in the presence or absence of $100 \mu \mathrm{M}$ IDO inhibitor, 1-methyl-DL-tryptophan (1-MT; Sigma-Aldrich) or $30 \mu \mathrm{M}$ p53 inhibitor, pifithrin- $\alpha$ (PFT; Santa Cruz Biotechnology, Inc., Dallas, TX, USA). The concentrations of 1-MT and PFT were selected, according to previous experiments that demonstrated efficacy without toxicity $(1,16,20,22)$. Pifithrin- $\alpha$ was refreshed in the cell cultures at day 4 . A total of $5 \times 10^{4}$ PBMCs from each member of the MLR couple were used, with a total of $1 \times 10^{5}$ PBMCs in each well. Cultures of resting PMBCs with a population of $1 \times 10^{5}$ cells/well were used as the control.

At the end of the 7-day period, cell proliferation was assessed using a cell proliferation enzyme-linked immunosorbent assay (ELISA; Roche Diagnostics, Basel, Switzerland) using bromodeoxyuridine labeling and immunoenzymatic detection according to the manufacturer's protocol. The proliferation index was calculated as the ratio of the optical density (OD) derived from each MLR to the mean of the ODs derived from the control resting PBMC cultures of the two subjects that constituted the specific MLR. The following formula was used: Proliferative index $=\mathrm{OD}$ of the MLR from subjects $A$ and $B /\{[(O D$ of resting $P B M C s$ from subject $\mathrm{A}+\mathrm{OD}$ of resting PBMCs from subject $\mathrm{B})] / 2\}$. A total of 10 MLRs were performed. All experiments were performed in triplicate, and the results presented are the mean of the three measurements.

Isolation of T-cells from MLRs and assessment of $\zeta$-chain, c-Myc,p53,p21, LDH-A and GLS2 levels. A total of $10 \mathrm{MLRs}$ were performed in 12-well plates for 7 days. The number of PBMCs from each member of the MLR couple was $5 \times 10^{5}$, with a total of $1 \times 10^{6} \mathrm{PBMCs} /$ well. The expression levels of ל-chain, c-Myc, p53, p21, LDH-A and GLS2 were assessed in the presence or absence of $100 \mu \mathrm{M} 1-\mathrm{MT}$ or $30 \mu \mathrm{M}$ PFT. PFT was refreshed in the cell cultures on day 4. Following the 7 day culture period, the T-cells were isolated by negative selection using the Pan T-cell Isolation kit (Miltenyi Biotec $\mathrm{GmbH})$.

Isolated T-cells were counted using an optical microscope and a Neubauer chamber, and cell viability was determined by trypan blue staining (Sigma-Aldrich). Equal numbers of T-cells from each MLR were lyzed using the T-PER tissue protein extraction reagent (Thermo Fisher Scientific, Inc., Waltham, MA, USA), supplemented with protease and phosphatase inhibitors (Sigma-Aldrich and Roche Diagnostics, respectively). The protein was quantified using a Bradford assay (Sigma-Aldrich) and western blotting was performed. Equal quantities of protein extracts $(50 \mu \mathrm{g})$ from each sample were loaded for electrophoresis in precast 4-12\% gradient bis-tris polyacrylamide gels (Invitrogen; Thermo Fisher Scientific, Inc.). Subsequently, the proteins were transferred onto polyvinylidene difluoride (PVDF) membranes (Invitrogen; Thermo Fisher Scientific, Inc.). Blots were blocked in 5\% w/v non-fat dry milk (Regilait, Saint Martin Belle Roche, France) diluted in 1X Tris-buffered saline (Thermo Fisher Scientific, Inc.) supplemented with $0.1 \%$ Tween-20 (Sigma-Aldrich). The blots were then incubated with the primary antibodies at $4^{\circ} \mathrm{C}$ for $16 \mathrm{~h}$, followed by secondary antibody incubation (anti-rabbit immunoglobulin $\mathrm{G}$, horseradish peroxidase-linked antibody; Cell Signaling Technology, Inc., Danvers, MA, USA) for $30 \mathrm{~min}$ at room temperature. A pre-stained protein ladder (Invitrogen; Thermo Fisher Scientific, Inc.) was used as a marker. The bands were visualized by enhanced chemiluminescent detection using the LumiSensor Plus Chemiluminescent 
Horseradish Peroxidase Substrate kit (GenScript, Piscataway, NJ, USA) and analysis was performed using Image $\mathbf{J}$ software v 1.49 (National Institute of Health, Bethesda, MD, USA). For the reprobing of PVDF blots, the previous primary and secondary antibodies were removed using Restore Western Blot Stripping Buffer (Thermo Fisher Scientific, Inc.), according to the manufacturer's instructions. The PVDF membrane was then reused and western blotting resumed as described, using a different primary antibody.

The following primary antibodies, all raised in rabbits with specificity for humans, were used for western blotting: Anti-ร-chain (cat. no. sc-20919; dilution, 1/100; Santa Cruz Biotechnology, Inc.), anti-c-Myc (cat. no. 5605; dilution, 1/500; Cell Signaling Technology, Inc.), anti-p53 (cat. no. 9282; dilution, 1/500; Cell Signaling Technology, Inc.), anti-p21 (cat. no. 2947; dilution, 1/500; Cell Signaling Technology, Inc.), anti-LDH-A (cat no. 2012; dilution, 1/1,000; Cell Signaling Technology, Inc.), anti-GLS2 (cat no. AP17426PU-N; dilution, 1/100; Acris Antibodies, San Diego, CA, USA) and anti- $\beta$-actin (cat no. 4967; dilution, 1/2,500; Cell Signaling Technology, Inc.).

Stimulation of isolated T-cells with TRP. T-cells were isolated from PBMCs using the Pan T-cell Isolation kit (Miltenyi Biotec $\mathrm{GmbH}$ ). Isolated T-cells were counted using an optical microscope and a Neubauer chamber. Cell viability was assessed by trypan blue staining (Sigma-Aldrich).

T-cells were cultured in the presence or absence of anti-CD2, anti-CD3 and anti-CD28 conjugated beads, using the T-Cell activation/expansion kit (Miltenyi Biotec $\mathrm{GmbH}$ ) at a bead to cell ratio of 1:2. Stimulated T-cells were cultured in the presence or absence of TRP $(0.25 \mathrm{mM})$. The concentration of TRP was selected according to previous studies that demonstrated efficacy without toxicity $(1,20)$.

Investigation of the effect of TRP on the proliferation of T-cells. T-cell proliferation was assessed using a Cell Proliferation ELISA (Roche Diagnostics). Resting, stimulated or stimulated in the presence of $0.25 \mathrm{mM}$ TRP T-cells were cultured in 96-well plates $\left(1 \times 10^{5}\right.$ cells/well) for $72 \mathrm{~h}$. All experiments were performed in T-cells derived from the blood of 10 individuals in triplicate, and the results are presented as the mean of the three measurements.

Assessment of the effect of TRP on $\zeta$-chain, $c-M y c, p 53$, p21, LDH-A and GLS2 levels in T-cells. The proteins were extracted from resting, stimulated or stimulated TRP-treated T-cells cultured in 12-well plates $\left(1 \times 10^{6}\right.$ cells/well) for $12 \mathrm{~h}$ in order to measure the expression levels of $\zeta$-chain, c-Myc, p53, p21, LDH-A and GLS2 by western blotting. The primary antibodies were anti- $\zeta$-chain (Santa Cruz Biotechnology, Inc.), anti-c-Myc (Cell Signaling Technology, Inc.), anti-p53 (Cell Signaling Technology, Inc.), anti-p21 (Cell Signaling Technology, Inc.), anti-LDH-A (Cell Signaling Technology, Inc.), anti-GLS2 (Acris Antibodies) and anti- $\beta$-actin (Cell Signaling Technology, Inc.). Experiments were performed in T-cells derived from the blood of 10 individuals.

Statistical analysis. Normality of the evaluated variables was assessed and confirmed by one-sample Kolmogorov-Smirnov

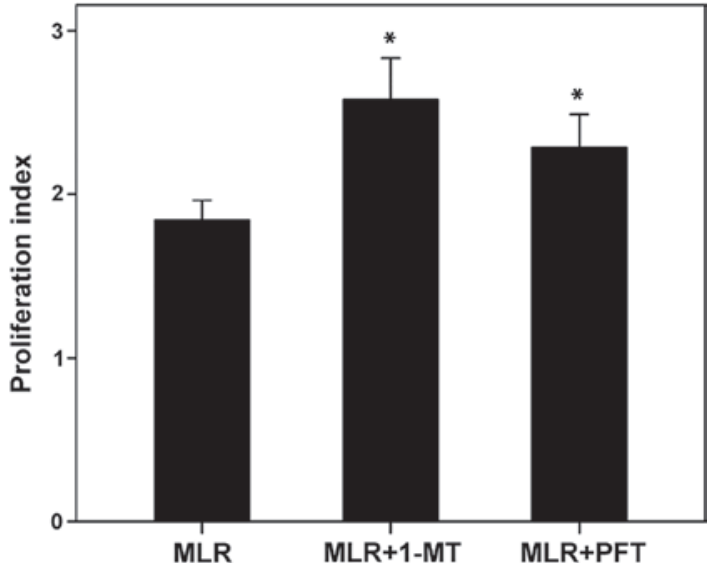

Figure 1. Inhibition of IDO or p53 increases cellular proliferation in MLRs. In MLRs, the IDO inhibitor 1-MT increases the proliferation index from $1.84 \pm 0.15$ to $2.57 \pm 0.30$ and the p53 inhibitor PFT from $1.84 \pm 0.15$ to $2.29 \pm 0.24$. Values are presented as the mean $\pm 95 \%$ confidence intervals. ${ }^{*} \mathrm{P}<0.05$ vs. untreated MLR. IDO, indoleamine 2,3-dioxygenase; MLRs, mixed lymphocyte reactions; 1-MT, 1-methyl-DL-tryptophan; PFT, pifithrin- $\alpha$.

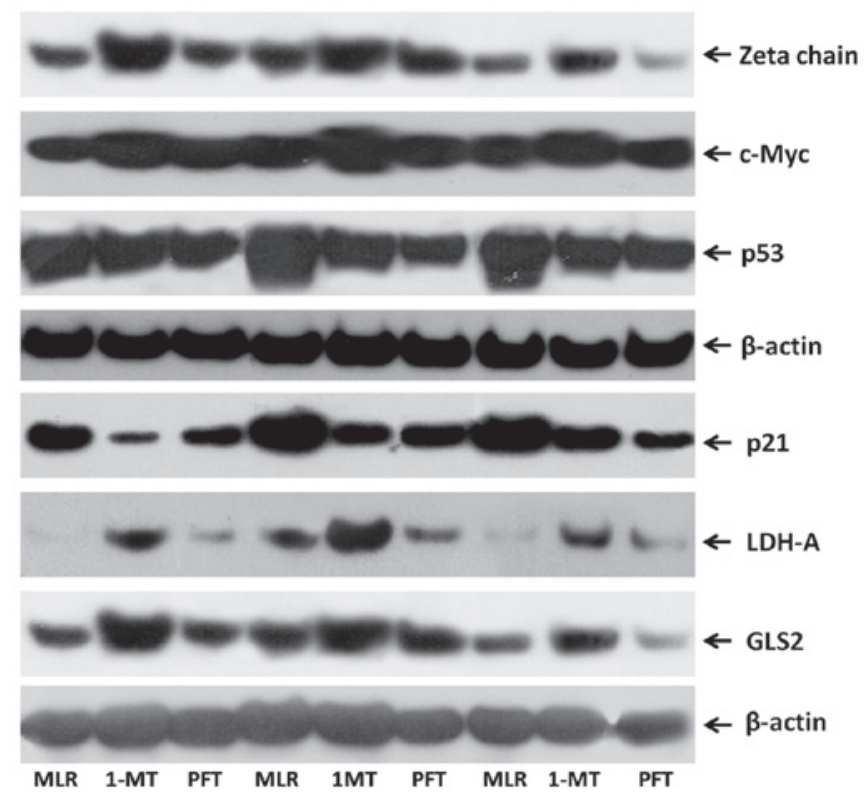

Figure 2. Western blot images presenting the effect of 1-MT or PFT treatment of MLRs on the expression levels of TCR-complex $\zeta$-chain, c-Myc, p53, p21, LDH-A and GLS2 in alloreactive T-cells. Ten MLRs were conducted in the presence or absence of the indoleamine 2,3-dioxygenase inhibitor 1-MT and the p53 inhibitor PFT, following which the T-cells were isolated and western blotting conducted. The western blotting lanes correspond to three representative experiments of the ten conducted. 1-MT, 1-methyl-DL-tryptophan; PFT, pifithrin- $\alpha$; MLRs, mixed lymphocyte reactions; TCR, T-cell receptor; LDH-A, lactose dehydrogenase A; GLS2, glutaminase 2.

test. For comparison of means, the sphericity assumption was evaluated by Mauchly's test and if it failed, degrees of freedom were corrected using Greenhouse-Geisser or Huynh-Feldt estimates of sphericity. Comparison of means was performed by one-way repeated-measures analysis of variance followed by Bonferroni's correction test. The values were normalized against the control group, and are presented as the mean \pm standard deviation. SPSS 13.0 for Windows (SPSS Inc., Chicago, IL, USA) was used for all statistical 
A

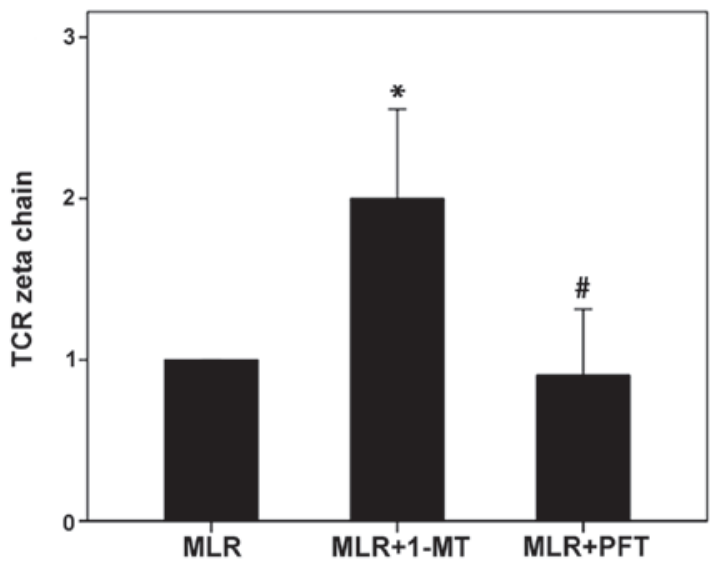

C

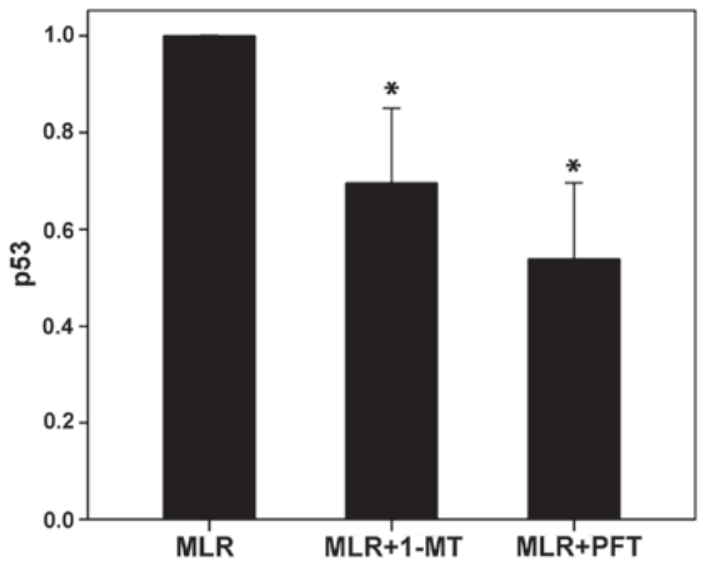

$\mathbf{E}$

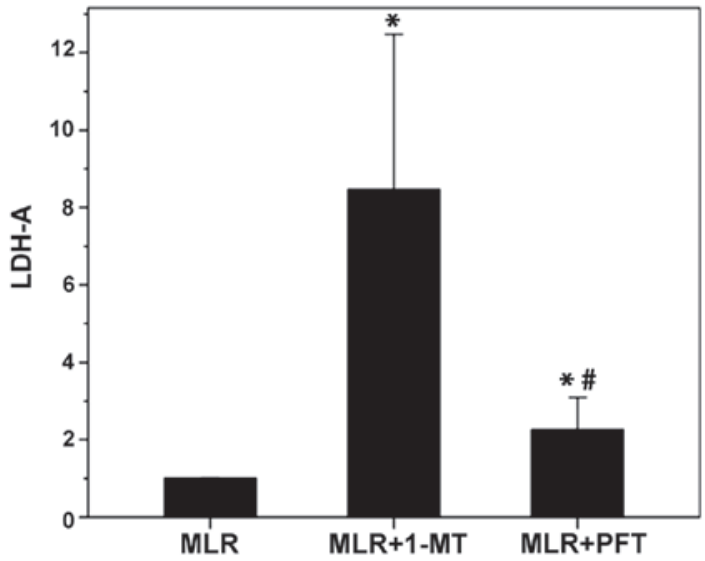

B

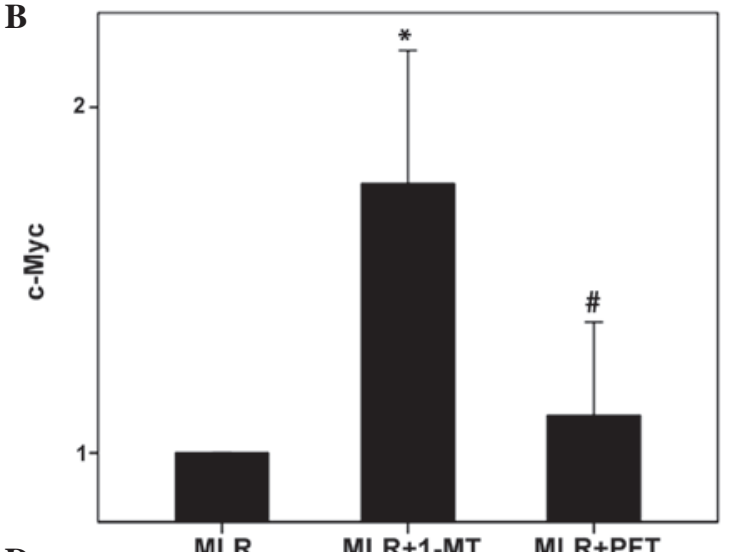

D

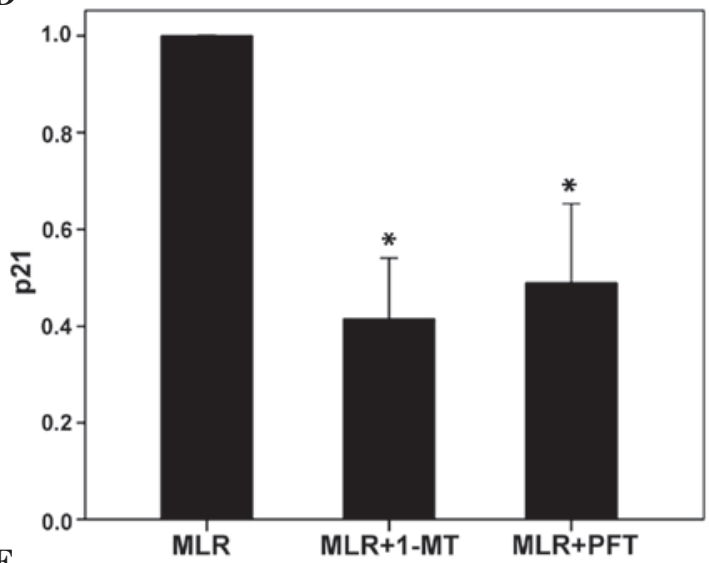

F

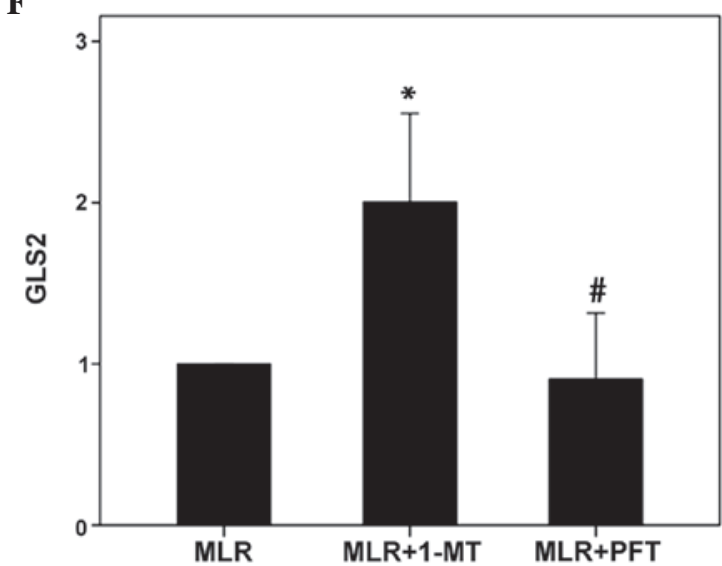

Figure 3. The effect of 1-MT or PFT treatment of MLRs on the expression levels of TCR-complex $\zeta$-chain, c-Myc, p53, p21, LDH-A and GLS2 in alloreactive T-cells. Ten MLRs were conducted in the presence or absence of the indoleamine 2,3-dioxygenase inhibitor 1-MT or the p53 inhibitor PFT, following which the T-cells were isolated and western blotting conducted. (A) 1-MT however not PFT increased TCR-complex $\zeta$-chain expression. (B) 1-MT however not PFT increased c-Myc expression. 1-MT and PFT reduced (C) p53 expression and (D) p21 expression. (E) 1-MT markedly induced LDH-A expression, whereas PFT increased it to a lesser extent. (F) 1-MT significantly increased GLS2 expression, while PFT did not affect the expression levels. Values are presented as the mean $\pm 95 \%$ confidence intervals. ${ }^{*} \mathrm{P}<0.05$ vs. untreated MLR; " $\mathrm{P}<0.05$ vs. 1 -MT-treated MLR. 1-MT, 1 -methyl-DL-tryptophan; PFT, pifithrin- $\alpha$; MLRs, mixed lymphocyte reactions; TCR, T-cell receptor; LDH-A, lactose dehydrogenase A; GLS2, glutaminase 2.

analyses. $\mathrm{P}<0.05$ was considered to indicate a statistically significant difference.

\section{Results}

IDO and p53 reduce T-cell proliferation. In MLRs, the inhibition of IDO by 1-MT enhanced the T-cell proliferation index from $1.84 \pm 0.15$ to $2.57 \pm 0.30(\mathrm{P}<0.001)$. In addition, $\mathrm{p} 53$ inhibition by PFT enhanced the T-cell proliferation index to $2.29 \pm 0.24$ $(\mathrm{P}<0.001)$. The proliferation index was not significantly different between the 1-MT or PFT treatment groups ( $\mathrm{P}=0.55$; Fig. 1).
IDO induces the expression levels of $p 53$ and $p 21$, however IDO alone reduces the expression levels of $\zeta$-chain, $c-M y c, L D H-A$ and GLS2.In MLRs, IDO inhibition reduced the expression levels of p53 and p21 in T-cells. In T-cells derived from 1-MT-treated MLRs, the expression levels of p53 were reduced to $0.70 \pm 0.15$, compared with the level in untreated MLRs $(\mathrm{P}=0.004$; Figs. 2 and 3). Similarly, following treatment with 1-MT, the expression of p21 was significantly reduced compared with the untreated MLRs $(0.41 \pm 0.12 ; \mathrm{P}<0.001)$. In addition, PFT reduced the expression levels of p53 and p21 to $0.54 \pm 0.15(\mathrm{P}=0.001)$ and $0.49 \pm 0.16(\mathrm{P}<0.001)$, respectively (Figs. 2 and 3$)$. 
In MLRs, IDO, however not p53, reduced the expression levels of $\zeta$-chain, c-Myc, LDH-A and GLS2. Compared with untreated MLRs, in 1-MT-treated T-cells, the expression of $\xi$-chain increased to $2.0 \pm 0.53(\mathrm{P}=0.006)$, c-Myc to $1.78 \pm 0.37(\mathrm{P}=0.003), \mathrm{LDH}-\mathrm{A}$ to $8.47 \pm 8.05(\mathrm{P}=0.001)$ and GLS2 to $2.0 \pm 0.52$ ( $\mathrm{P}=0.005$; Figs. 2 and 3 ).

Compared with untreated MLRs, in the PFT-treated T-cells the expression levels of $\zeta$-chain, c-Myc and GLS2 were unaltered, with levels of $0.90 \pm 0.16(\mathrm{P}=0.573), 1.11 \pm 0.26(\mathrm{P}=$ $0.348)$ and $0.90 \pm 0.16(\mathrm{P}=0.577)$, respectively. PFT increased the expression of $\mathrm{LDH}-\mathrm{A}$ to $2.27 \pm 1.67(\mathrm{P}=0.05)$, however, to a lesser extent compared with 1-MT, which increased LDH-A to $8.47 \pm 8.05(\mathrm{P}=0.001$; Figs. 2 and 3$)$.

In activated $T$-cells, TRP reduces $T$-cell proliferation. In T-cells activated with anti-CD2, anti-CD3 and anti-CD28, TRP reduced proliferation. The proliferation index was $5.04 \pm 0.96$ in activated T-cells and $2.91 \pm 0.42$ in activated T-cells treated with TRP ( $\mathrm{P}<0.001 ;$ Fig. 4$)$.

In activated T-cells, TRP induces the expression levels of p53 and p21, while reducing the expression levels of $\zeta$-chain, $c-M y c, L D H-A$ and $G L S 2$. Direct activation of the GCN2 kinase by TRP induced the expression levels of p53 and p 21 in activated T-cells. Compared with the unactivated control T-cells, p53 levels were increased in activated T-cells to $3.56 \pm 1.83(\mathrm{P}<0.001)$, with TRP-treated activated T-cells exhibiting a further increase to $5.2 \pm 2.24(\mathrm{P}<0.001)$. It is noteworthy that in the absence of TRP, activation of T-cells increased the levels p53, however, to a significantly lesser extent $(\mathrm{P}<0.001$; Figs. 5 and 6).

Similar results were observed regarding the expression of p21. Compared with the unactivated T-cells, TRP-treated activated T-cells exhibited an increase in the expression of p21 to $5.05 \pm 5.76(\mathrm{P}=0.002)$, whereas in untreated activated T-cells, p21 levels were increased to $3.47 \pm 3.78(\mathrm{P}=0.004)$. It is noteworthy that in the absence of TRP, activation of T-cells increased the expression of $\mathrm{p} 21(\mathrm{P}=0.001)$ however, to a significantly lesser extent (Figs. 5 and 6).

Activation of T-cells resulted in an almost 3-fold increase in $\zeta$-chain expression $(2.81 \pm 1.30 ; \mathrm{P}=0.06)$. However, treatment of activated T-cells with TRP reduced the levels of $\zeta$-chain to $0.88 \pm 0.07$ ( $\mathrm{P}=0.02$; Figs. 5 and 6 ).

Similar results were observed regarding the expression of c-Myc. Activation of T-cells resulted in an increase in c-Myc expression to $2.32 \pm 1.60(\mathrm{P}=0.045)$. However, treatment of activated T-cells with TRP reduced the levels of c-Myc to $0.67 \pm 0.40$ ( $\mathrm{P}=0.040$; Figs. 5 and 6$)$.

Compared with unactivated T-cells, T-cell activation with anti-CD2, anti-CD3 and anti-CD28 increased the expression of LDH-A 3-fold (2.98 $\pm 2.60 ; \mathrm{P}=0.008)$ and GLS2 2-fold (2.19 $\pm 0.77 ; \mathrm{P}=0.003)$. Concurrent treatment with TRP abolished these alterations in the expression of LDH-A and GLS2, with levels of $0.94 \pm 0.39(\mathrm{P}=0.523)$ and $1.18 \pm 0.69(\mathrm{P}=0.482)$, respectively (Figs. 5 and 6).

\section{Discussion}

IDO suppresses T-cell proliferation and concurrently inhibits aerobic glycolysis and glutaminolysis $(16,20)$. In addition, IDO increases the levels of p53, which has been suggested to

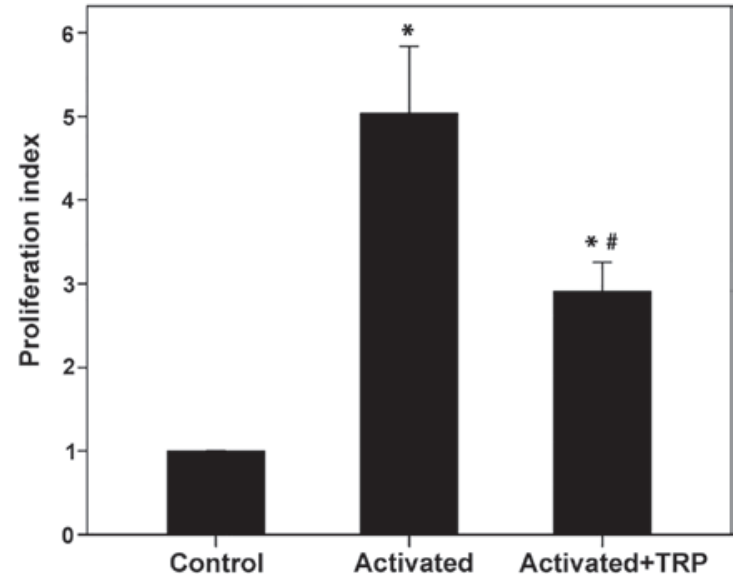

Figure 4. The effect of TRP on the proliferation of activated T-cells. In T-cells activated with anti-CD2, anti-CD3 and anti-CD28, TRP reduced proliferation. The proliferation index was $5.04 \pm 0.96$ in activated T-cells and $2.91 \pm 0.42$ in activated T-cells treated with TRP. Values are presented as the mean $\pm 95 \%$ confidence intervals. ${ }^{*} \mathrm{P}<0.05$ vs. unactivated control T-cells; ${ }^{\#} \mathrm{P}<0.05$ vs. activated T-cells. TRP, tryptophanol; $\mathrm{CD}$, cluster of differentiation.

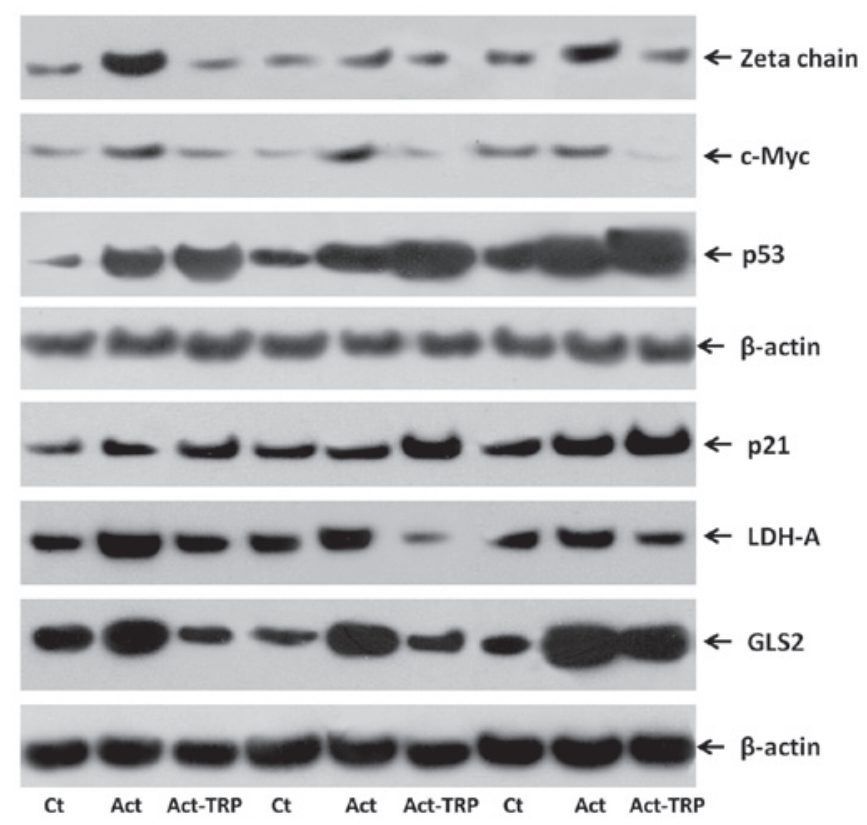

Figure 5. Western blot images presenting the effect of TRP treatment on the expression levels of TCR-complex $\zeta$-chain, c-Myc, p53, p21, LDH-A and GLS2 in activated T-cells. Isolated T-cells were resting or activated with anti-CD2, anti-CD3 and anti-CD28 in the presence or absence of the GCN2 kinase activator, TRP. The western blotting lanes correspond to three representative experiments of the ten conducted. TRP, tryptophanol; TCR, T-cell receptor; LDH-A, lactose dehydrogenase A; GLS2, glutaminase 2; $\mathrm{CD}$, cluster of differentiation; GCN2, general control nonderepressible 2; Ct, control; Act, activated.

contribute to reduced T-cell proliferation and downregulation of various factors involved in aerobic glycolysis. Notably, IDO-induced increases in the levels of p53 do not alter LDH-A and GLS2 levels (20). In addition, IDO downregulates TCR-complex $\zeta$-chain (23). Of the ten immunoreceptor tyrosine activation motifs (ITAMs) of the TCR-complex, six are within the $\zeta$-chain dimer. Reduced phosphorylation of TCR-complex ITAMs results in reduced c-Myc expression and subsequently reduced T-cell proliferation (21). In addition, upon T-cell 
A

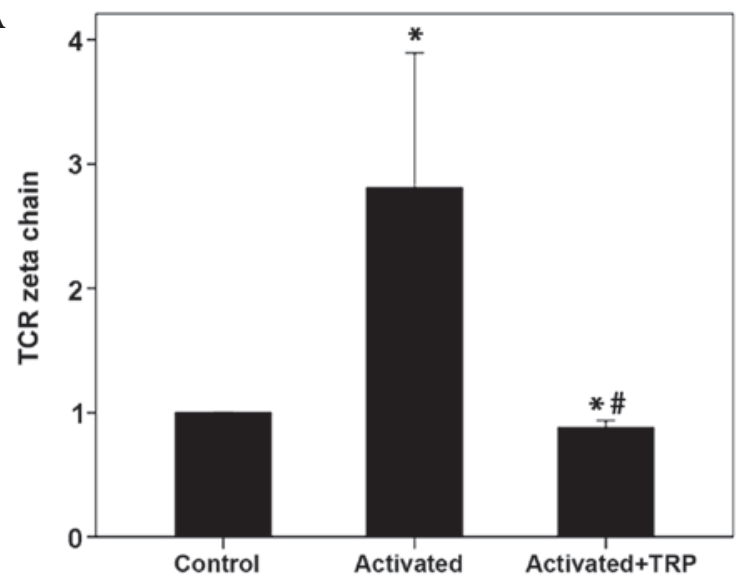

C

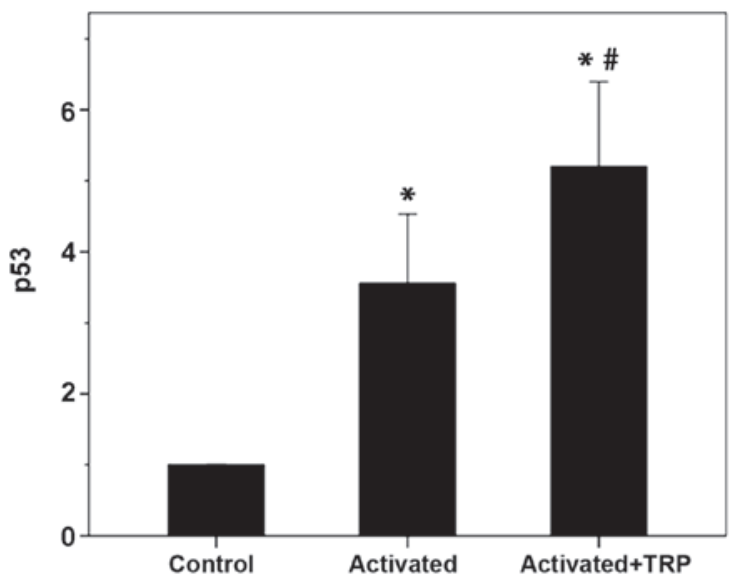

$\mathbf{E}$

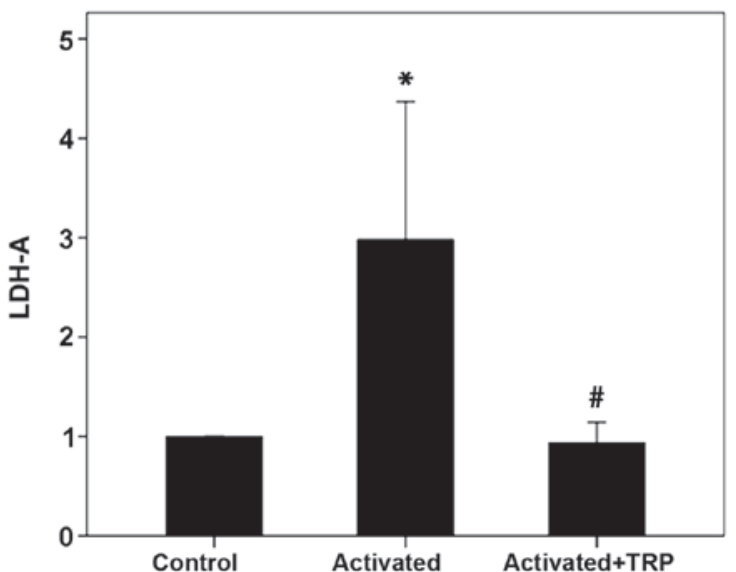

B

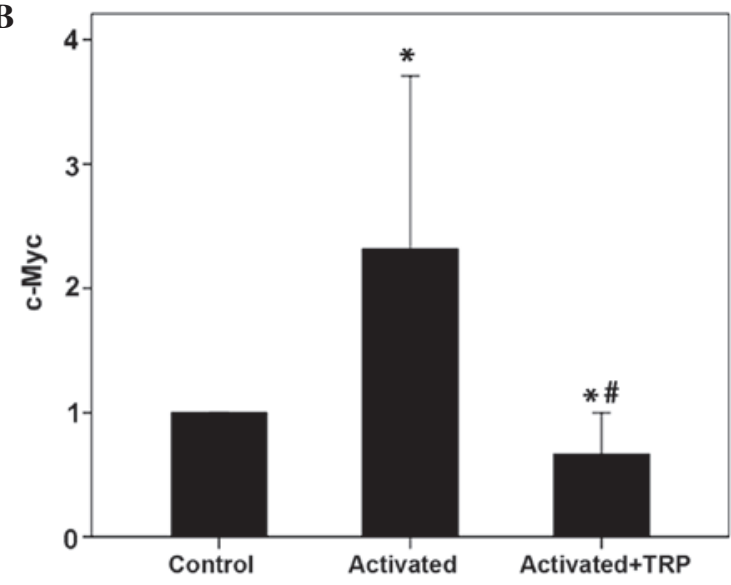

D

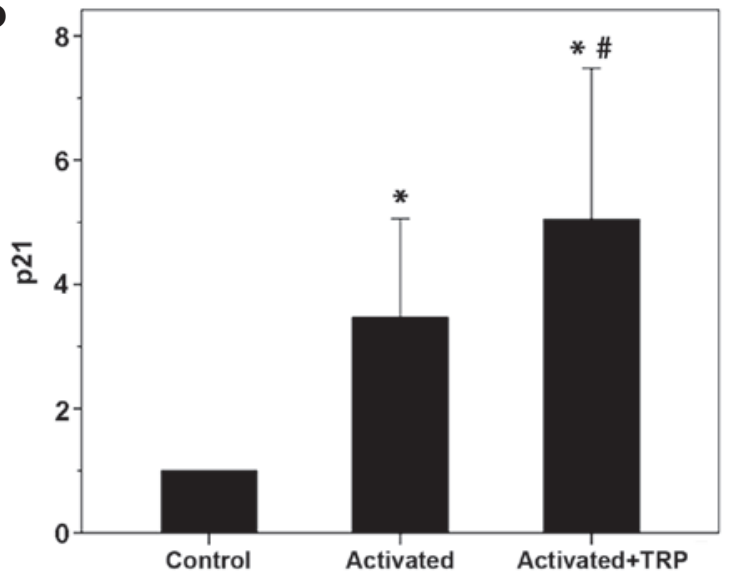

$\mathbf{F}$

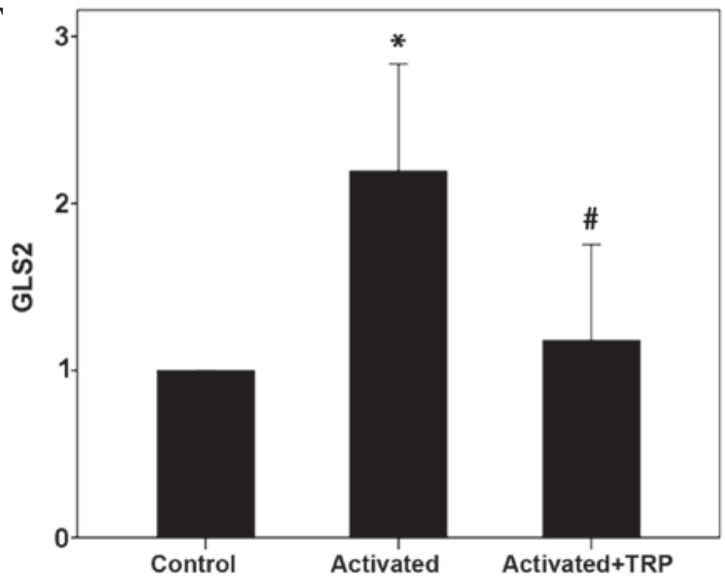

Figure 6. The effect of TRP treatment on the expression levels of TCR-complex $\zeta$-chain, c-Myc, p53, p21, LDH-A and GLS2 in activated T-cells. Isolated T-cells were resting or activated with anti-CD2, anti-CD3 and anti-CD28 in the presence or absence of the GCN2 kinase activator TRP. Ten experiments were conducted. (A) T-cell activation increased TCR-complex $\xi$-chain expression, whereas treatment with TRP reduced expression. (B) T-cell activation increased c-Myc expression, whereas treatment with TRP reduced expression. (C) T-cell activation increased p53 expression levels, which were further increased following TRP treatment. (D) T-cell activation increased the expression of p21, which was further increased by TRP treatment. (E) Activation of T-cells increased LDH-A expression, whereas treatment with TRP reduced expression. (F) The expression levels of GLS2 were increased in activated T-cells however were reduced following TRP treatment. Values are presented as the mean $\pm 95 \%$ confidence intervals. ${ }^{~} \mathrm{P}<0.05$ vs. unactivated control T-cells; ${ }^{*} \mathrm{P}<0.05$ vs. activated T-cells. TRP, tryptophanol; TCR, T-cell receptor; LDH-A, lactose dehydrogenase A; GLS2, glutaminase 2; CD, cluster of differentiation; GCN2, general control nonderepressible 2 .

activation, c-Myc is upregulated and induces the expression levels of LDH-A and GLS2 (19). In the current study, it was investigated whether IDO-induced L-tryptophan depletion or direct GCN2 kinase activation promote the following sequence of events: TCR-complex $\xi$-chain downregulation, reduced c-Myc expression, reduced T-cell proliferation and downregulation of LDH-A and GLS2 levels. In addition, the effect of IDO or direct GCN2 kinase activation on p53 expression was investigated.
For the purposes of the present study, the MLR as a model of alloreactivity was used (24), in addition to the specific IDO inhibitor 1-MT. 1-MT is a competitive, non-toxic IDO inhibitor (25), which has been successfully used to breach the immune privilege of the placenta and tolerance against grafts $(5,11)$. In addition, the p53 inhibitor PFT was used to investigate the association between $\mathrm{p} 53$ and c-Myc, and to confirm the p53-independent effects of IDO on the expression 
levels of LDH-A and GLS2. PFT acts downstream of p53 and reversibly inhibits p53-dependent transcriptional activation (22). Furthermore, a system lacking IDO-bearing APCs was used in order to distinguish the effect of GCN2 kinase activation from a possible effect of kynurenine, and to investigate whether this activation is adequate to induce the observed alterations by IDO, independently of mTORC1. Isolated T-cells were activated with anti-CD2, anti-CD3 and anti-CD28 antibodies in the presence or absence of TRP. TRP is a competitive inhibitor of the tryptophanyl-tRNA synthetase. By raising the pool of uncharged tRNA, TRP acts as a pharmacologic activator of the GCN2 kinase (26). Notably, halofuginone, which activates the GCN2 kinase, exerts its immunomodulatory properties without altering signaling through the mTORC1 (27).

The present study demonstrated that in MLRs, the IDO inhibitor, 1-MT, increased T-cell proliferation, indicating that IDO reduces proliferation. Furthermore, the p53 inhibitor, PFT, increased proliferation, which indicated that p53 inhibits proliferation in MLRs. In addition to the activation of the GCN2 kinase, the immunomodulatory effects of IDO have been attributed to kynurenine, the first breakdown product in the IDO-dependent tryptophan degradation pathway. Kynurenine is able to affect T-cells by activating the aryl hydrocarbon receptor $(28,29)$. In order to elucidate whether GCN2 activation alone is adequate for suppressing T-cell proliferation, the current study used a kynurenine free, APC-free system of isolated T-cell activation to investigate the effects of the GCN2 kinase activator, TRP. This demonstrated that in activated T-cells, TRP inhibited T-cell proliferation.

As observed in a previous study (30), IDO induced the expression levels of p53 and p21 in MLR-derived T-cells, contributing to reduced $\mathrm{T}$-cell proliferation due to the p53-mediated upregulation of $\mathrm{p} 21$, the latter being a potent cyclin-dependent kinase inhibitor, which induces $\mathrm{G}_{1}$-phase cell-cycle arrest (31). The reduction in the expression levels of p53 and p21 in PFT-treated alloreactive T-cells indicates that PFT potentially downregulates a positive feedback loop that controls the expression of p53 in these cells (32).

The present study demonstrated that direct activation of the GCN2 kinase by TRP in T-cells activated with anti-CD2, anti-CD3 and anti-CD28 markedly increased p53 and p21 expression, suggesting that activation of this kinase alone is sufficient for these alterations. Notably, compared with the resting control T-cells, in activated T-cells p53 and p21 levels were increased, however to a lesser extent compared with in the TRP-treated activated T-cells. This may be an intrinsic cell mechanism for controlling proliferation. For instance, in primary embryonic fibroblasts, c-Myc, a transcription factor that is required for cell proliferation, activates the $\mathrm{p} 19^{\mathrm{ARF}}$-mouse double minute 2 homolog-p53 tumor suppressor pathway (33).

Furthermore, in accordance with a previous study (30), IDO reduced LDH-A and GLS2 levels in MLR-derived T-cells. The p53 inhibitor, PFT, revealed no effect on GLS2 expression and exerted a relatively minor effect on the LDH-A levels. In the present study, direct GCN2 kinase activation by TRP in T-cells activated with anti-CD2, anti-CD3 and anti-CD28 reduced LDH-A and GLS2 levels indicating that GCN2 kinase activation alone is sufficient to induce downregulation of the enzymes involved in aerobic glycolysis and glutaminolysis, respectively. Since, upon activation, rapidly proliferating $\mathrm{T}$ cells rely on aerobic glycolysis and glutaminolysis in order to fulfill their bioenergetic and biosynthetic demands (19), this p53- and kynurenine-independent downregulation of LDH-A and GLS2, respectively, by IDO may contribute to its immunosuppressive effects.

In MLRs, IDO reduced the levels of TCR-complex $\zeta$-chain and c-Myc, whereas PFT had no effect, indicating that p53 does not effect the levels of $\zeta$-chain and c-Myc. The reduction in c-Myc may be attributed to the reduced levels of $\zeta$-chain. It is known that reduced phosphorylation of the TCR complex ITAMs downregulates c-Myc expression and inhibits T-cell proliferation (21). Therefore, beyond the IDO-induced increase in p53, the IDO-induced decrease in c-Myc may additionally contribute to the IDO-induced inhibition of T-cell proliferation. Similar results were obtained by TRP treatment of isolated activated T-cells, indicating that the GCN2 kinase alone is sufficient for the downregulation of TCR-complex $\zeta$-chain and c-Myc. It is noteworthy that the levels of TCR-complex $\zeta$-chain were increased in activated T-cells in contrast with resting T-cells, although the responsible mechanisms remain to be elucidated.

The results of the present study supported the notion that in primary human T-cells, IDO reduces LDH-A levels through the downregulation of c-Myc. LDH-A, which converts pyruvate to lactate in the last step of aerobic glycolysis, is a putative c-Myc target gene. Transgenic mice overexpressing c-Myc in the liver exhibit increased hepatic glycolytic enzyme activity and overproduce lactate (34). In addition, transfected rodent fibroblasts overexpressing LDH-A alone, or those transformed by c-Myc overproduce lactate (35). Furthermore, in activated murine T-cells, c-Myc is upregulated and induces LDH-A expression (19).

Upregulation of c-Myc in activated mouse T-cells has been demonstrated to increase the levels of GLS2 (19). In addition to rapidly proliferating T-cells, numerous rapidly proliferating cells, notably cancer cells, reprogram their mitochondrial metabolism to depend on glutaminolysis to sustain cellular viability and Krebs cycle anapleurosis. In transformed cells, overexpression of c-Myc results in the concurrent conversion of glucose to lactate and the oxidation of glutamine via the Krebs cycle (36). In this study, in human T-cells, IDO-induced GLS2 downregulation may be mediated through the reduction in c-Myc expression.

Thus, in the present study, by using a single experimental model it was possible to confirm the observations of previous studies, which used diverse experimental models. In addition, the present study provided further insight into the mechanism potentially responsible for the immunosuppressive effects of IDO. More precisely, these data demonstrated that IDO, through GCN2 kinase activation, downregulates TCR-complex $\zeta$-chain and $\mathrm{c}-\mathrm{Myc}$, resulting in the suppression of T-cell proliferation and reduction in the levels of LDH-A and GLS2, which are key enzymes involved in aerobic glycolysis and glutaminolysis, respectively.

\section{References}

1. Munn DH, Sharma MD, Baban B, Harding HP,Zhang Y, Ron D and Mellor AL: GCN2 kinase in T cells mediates proliferative arrest and anergy induction in response to indoleamine 2,3-dioxygenase. Immunity 22: 633-642, 2005. 
2. Cobbold SP, Adams E, Farquhar CA, Nolan KF, Howie D, Lui KO, Fairchild PJ, Mellor AL, Ron D and Waldmann H: Infectious tolerance via the consumption of essential amino acids and mTOR signaling. Proc Natl Acad Sci USA 106: 12055-12060, 2009.

3. King NJ and Thomas SR: Molecules in focus: Indoleamine 2,3-dioxygenase. Int J Biochem Cell Biol 39: 2167-2172, 2007.

4. Curti A, Trabanelli S, Salvestrini V, Baccarani M and Lemoli RM: The role of indoleamine 2,3-dioxygenase in the induction of immune tolerance: Focus on hematology. Blood 113 : 2394-2401, 2009.

5. Alexander AM, Crawford M, Bertera S, Rudert WA, Takikawa O, Robbins PD and Trucco M: Indoleamine 2,3-dioxygenase expression in transplanted NOD Islets prolongs graft survival after adoptive transfer of diabetogenic splenocytes. Diabetes 51: 356-365, 2002

6. Beutelspacher SC, Pillai R, Watson MP, Tan PH, Tsang J, McClure MO, George AJ and Larkin DF: Function of indoleamine 2,3-dioxygenase in corneal allograft rejection and prolongation of allograft survival by over-expression. Eur J Immunol 36: 690-700, 2006

7. Li Y, Tredget EE, Ghaffari A, Lin X, Kilani RT and Ghahary A: Local expression of indoleamine 2,3-dioxygenase protects engraftment of xenogeneic skin substitute. J Invest Dermatol 126 128-136, 2006

8. Seo SK, Choi JH, Kim YH, Kang WJ, Park HY, Suh JH, Choi BK, Vinay DS and Kwon BS: 4-1BB-mediated immunotherapy of rheumatoid arthritis. Nat Med 10: 1088-1094, 2004.

9. Gurtner GJ, Newberry RD, Schloemann SR, McDonald KG and Stenson WF: Inhibition of indoleamine 2,3-dioxygenase augments trinitrobenzene sulfonic acid colitis in mice. Gastroenterology 125: 1762-1773,2003.

10. Kwidzinski E, Bunse J, Aktas O, Richter D, Mutlu L, Zipp F, Nitsch R and Bechmann I: Indolamine 2,3-dioxygenase is expressed in the CNS and down-regulates autoimmune inflammation. FASEB J 19: 1347-1349, 2005.

11. Munn DH, Zhou M, Attwood JT, Bondarev I, Conway SJ, Marshall B, Brown C and Mellor AL: Prevention of allogeneic fetal rejection by tryptophan catabolism. Science 281: 1191-1193, 1998.

12. Mellor AL, Sivakumar J, Chandler P, Smith K, Molina H, Mao D and Munn DH: Prevention of T cell-driven complement activation and inflammation by tryptophan catabolism during pregnancy. Nat Immunol 2: 64-68, 2001.

13. Munn DH and Mellor AL: Indoleamine 2,3-dioxygenase and tumor-induced tolerance. J Clin Invest 117: 1147-1154, 2007.

14. Eleftheriadis T, Liakopoulos V, Antoniadi G, Stefanidis I and Galaktidou G: Indoleamine 2,3-dioxygenase is increased in hemodialysis patients and affects immune response to hepatitis B vaccination. Vaccine 29: 2242-2247, 2011.

15. Eleftheriadis T, Yiannaki E, Antoniadi G, Liakopoulos V, Pissas G, Galaktidou G and Stefanidis I: Plasma indoleamine 2,3-dioxygenase and arginase type I may contribute to decreased blood T-cell count in hemodialysis patients. Ren Fail 34: 1118-1122, 2012

16. Eleftheriadis T, Pissas G, Yiannaki E, Markala D, Arampatzis S, Antoniadi G, Liakopoulos V and Stefanidis I: Inhibition of indoleamine 2,3-dioxygenase in mixed lymphocyte reaction affects glucose influx and enzymes involved in aerobic glycolysis and glutaminolysis in alloreactive T-cells. Hum Immunol 74: 1501-1509, 2013

17. Eleftheriadis T, Pissas G, Karioti A, Antoniadi G, Liakopoulos V, Dafopoulou K, Pournaras S, Koukoulis G and Stefanidis I: The indoleamine 2,3-dioxygenase inhibitor 1-methyl-tryptophan suppresses mitochondrial function, induces aerobic glycolysis and decreases interleukin-10 production in human lymphocytes. Immunol Invest 41: 507-520, 2012.

18. Warburg O: On the origin of cancer cells. Science 123: 309-314, 1956.

19. Wang R, Dillon CP, Shi LZ, Milasta S, Carter R, Finkelstein D, McCormick LL, Fitzgerald P, Chi H, Munger J and Green DR: The transcription factor Myc controls metabolic reprogramming upon T lymphocyte activation. Immunity 35: 871-882, 2011.
20. Eleftheriadis T, Pissas G, Antoniadi G, Spanoulis A, Liakopoulos V and Stefanidis I: Indoleamine 2,3-dioxygenase increases p53 levels in alloreactive human $\mathrm{T}$ cells and both indoleamine 2,3-dioxygenase and p53 suppress glucose uptake, glycolysis and proliferation. Int Immunol 26: 673-684, 2014.

21. Guy CS, Vignali KM, Temirov J, Bettini ML, Overacre AE, Smeltzer M, Zhang H, Huppa JB, Tsai YH, Lobry C, et al: Distinct TCR signaling pathways drive proliferation and cytokine production in T cells. Nat Immunol 14: 262-270, 2013.

22. Komarov PG, Komarova EA, Kondratov RV, Christov-Tselkov K, Coon JS, Chernov MV and Gudkov AV: A chemical inhibitor of p53 that protects mice from the side effects of cancer therapy. Science 285: 1733-1737, 1999

23. Fallarino F, Grohmann U, You S, McGrath BC, Cavener DR, Vacca C, Orabona C, Bianchi R, Belladonna ML, Volpi C, et al: The combined effects of tryptophan starvation and tryptophan catabolites down-regulate $\mathrm{T}$ cell receptor zeta-chain and induce a regulatory phenotype in naive T cells. J Immunol 176: 6752-6761, 2006.

24. Sato T, Deiwick A, Raddatz G, Koyama K and Schlitt HJ: Interactions of allogeneic human mononuclear cells in the two-way mixed leucocyte culture (MLC): Influence of cell numbers, subpopulations and cyclosporin. Clin Exp Immunol 115: 301-308, 1999

25. Jia L, Schweikart K, Tomaszewski J, Page JG, Noker PE, Buhrow SA, Reid JM, Ames MM and Munn DH: Toxicology and pharmacokinetics of 1-methyl-d-tryptophan: Absence of toxicity due to saturating absorption. Food Chem Toxicol 46: 203-211, 2008.

26. Jiang HY, Wek SA, McGrath BC, Scheuner D, Kaufman RJ, Cavener DR and Wek RC: Phosphorylation of the alpha subunit of eukaryotic initiation factor 2 is required for activation of NF-kappaB in response to diverse cellular stresses. Mol Cell Biol 23: 5651-5663, 2003

27. Sundrud MS, Koralov SB, Feuerer M, Calado DP, Kozhaya AE, Rhule-Smith A, Lefebvre RE, Unutmaz D, Mazitschek R, Waldner $\mathrm{H}$, et al: Halofuginone inhibits TH17 cell differentiation by activating the amino acid starvation response. Science 324 : 1334-1338, 2009

28. Mezrich JD, Fechner JH, Zhang X, Johnson BP, Burlingham WJ and Bradfield CA: An interaction between kynurenine and the aryl hydrocarbon receptor can generate regulatory $\mathrm{T}$ cells. J Immunol 185: 3190-3198, 2010.

29. Opitz CA, Litzenburger UM, Sahm F, Ott M, Tritschler I, Trump S, Schumacher T, Jestaedt L, Schrenk D, Weller M, et al: An endogenous tumour-promoting ligand of the human aryl hydrocarbon receptor. Nature 478: 197-203, 2011.

30. Elefheriadis T, Pissas G, Antoniadi G, Spanoulis A, Liakopoulos V and Stefanidis I: Indoleamine 2,3-dioxygenase increases p53 level in alloreactive human T-cells and both indoleamine 2,3-dioxygenase and p53 suppress glucose uptake, glycolysis and proliferation. Int Immunol 26: 673-684, 2014.

31. Brady CA and Attardi LD: p53 at a glance. J Cell Sci 123 (Pt 15): 2527-2532, 2010

32. Harris SL and Levine AJ: The p53 pathway: Positive and negative feedback loops. Oncogene 24: 2899-2908, 2005.

33. Eischen CM, Weber JD, Roussel MF, Sherr CJ and Cleveland JL: Disruption of the ARF-Mdm2-p53 tumor suppressor pathway in Myc-induced lymphomagenesis. Genes Dev 13: 2658-2669, 1999.

34. Valera A, Pujol A, Gregori X, Riu E, Visa J and Bosch F: Evidence from transgenic mice that myc regulates hepatic glycolysis. Faseb J 9: 1067-1078, 1995.

35. Shim H, Dolde C, Lewis BC, Wu CS, Dang G, Jungmann RA, Dalla-Favera R and Dang CV: c-Myc transactivation of LDH-A: Implications for tumor metabolism and growth. Proc Natl Acad Sci USA 94: 6658-6663, 1997.

36. Le A, Lane AN, Hamaker M, Bose S, Gouw A, Barbi J, Tsukamoto T, Rojas CJ, Slusher BS, Zhang H, et al: Glucose-independent glutamine metabolism via TCA cycling for proliferation and survival in B cells. Cell Metab 15: 110-121, 2012. 